\title{
Smartphone Ubiquitous Healthcare Diagnosis System
}

\author{
Eui-Bung Jeoung ${ }^{1}$, Soomi Hong ${ }^{2}$ and Kee-young Park ${ }^{2}$ \\ ${ }^{1}$ Automotive \& Mechanical Engineering, Howon University, Gunsan city \\ ${ }^{2}$ Public Health Administration, Digital Electronics \& Information, Jeonju Vision \\ University, Jeonju city, Korea \\ Bung4524@hanmail.net,smhong@jvision.ac.kr,kypark@jvision.ac.kr
}

\begin{abstract}
The modern day is witnessing rapid development of interest in health following the improvement of medical treatment technology as well as of the standard of life. Increasing demand for cutting-edge IT technology for health service is based on the desire better life. It changes the focus of health care service paradigm from treatmentoriented suppliers to consumer who requires preventive healthcare and wellness. Although widely distributed smart devices are used to provide smart health care service in order to keep pace with this paradigm change, it suffers from lack of integrated service system. The most universal touchstone to measure patient's state is body temperature, blood pressure, heart rate, oxygen saturation level, stethoscope, etc. Basic medical instruments are kept in many homes for family health. However, most personal medical equipment so far is stand-alone products which cannot keep tracks for family health issues. u-Healthcare system for smart phones uses vital-integrated communication module. This smart application for diagnosis system sends body sounds and signals (temperature, blood pressure, heart rate, oxygen saturation level, and stethoscope) to smart phone for diagnosis regardless of time and place. This study describes hardware construction of the diagnostic system in detail and argues new concept of diagnostic system environment via a smart phone application.
\end{abstract}

Keywords: VICM (Vital Integrated Communication Module), LCR (Level Crossing Rate), Diagnostic System, Vital Sound

\section{Introduction}

While the mobile phone has been widely used for several decades, smartphones are a more recent advance. Therefore, it is necessary to build which equipment consists of new idea and concept of vital integrated communication module suited for vital sounds data transfer purposes. In this paper, we'd like to show useful VICM (Vital Integrated Communication Module) diagnosis system for transfer of the vital sounds by smart App. Program. The VICM (Vital Integrated Communication Module) diagnosis system block diagram is shown in Figure 1. 


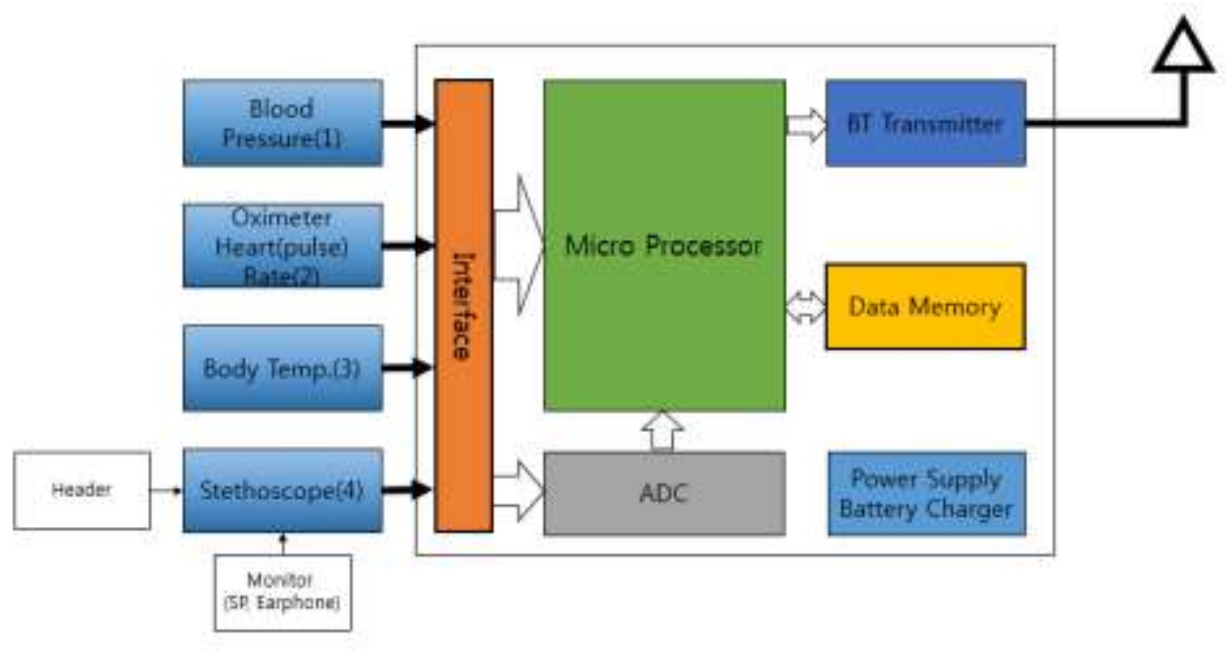

Figure 1. Vital Integrated Communication Module (VICM)

\section{Hardware Configuration and Function}

The VICM diagnosis system block diagram is shown in Figure 1. The VICM diagnosis system has been designed with sphygmomanometer, oximeter, body temperature and a stethoscope terminal and microprocessor controls the terminal. In other words, the biosounds data transmitted by four independent terminals is transmitted to the smartphone via wireless (BT: Bluetooth) communication under the control of the micro-processor. The transmitted bio-sounds data is displayed on smartphone window through the Apps. Also, the configuration and operation of the four terminals are as follows.

\subsection{Blood Pressure (BP)}

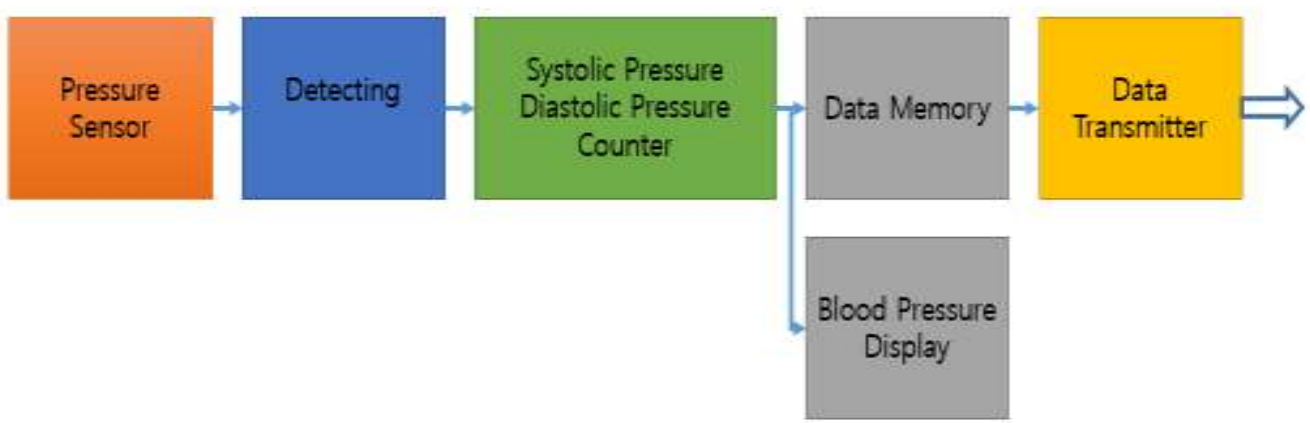

Figure 2. Blood Pressure Block Diagram

The Blood pressure (BP) block diagram is shown in Figure 2. Pressure sensor is detecting the blood vessels which are the parts of the circulatory system that transports blood throughout the human body. And blood pressure (BP) is the pressure of circulating blood on the walls of blood vessels. When used without further specification, "blood pressure" usually refers to the arterial pressure in the systemic circulation. Blood pressure is usually expressed in terms of the systolic (maximum during one heart beat) pressure over diastolic (minimum in between two heart beats) pressure and is measured in millimeters of mercury $(\mathrm{mmHg}$ ), above the surrounding atmospheric pressure (considered to be zero for convenience). Normal resting systolic (diastolic) blood pressure by counter in an adult is approximately $120 \mathrm{mmHg}(80 \mathrm{mmHg})$, abbreviated "120/80 $\mathrm{mmHg} "[1]$. 
BP data is saved in the data memory and displayed on sphygmomanometer. Also, the $\mathrm{BP}$ data is transmitted to the interface of microprocessor.

\subsection{Oximeter and Heart (Pulse) Rate}

Pulse oximetry is a noninvasive method for monitoring a person's oxygen saturation (SO2). Its reading of $\mathrm{SpO} 2$ (peripheral oxygen saturation) is not always identical to the reading of $\mathrm{SaO} 2$ (arterial oxygen saturation) from arterial blood gas analysis, but the two are correlated well enough that the safe, convenient, noninvasive, inexpensive pulse oximetry method is valuable for measuring oxygen saturation in clinical use. [1] A pair of LED is facing a photodiode through a translucent part of the patient's fingertip in the shown Figure 3. One LED is red, width wavelength of $660 \mathrm{~nm}$, and the other is infrared with a wavelength of $940 \mathrm{~nm}$. Absorption of light at these wavelengths differs significantly between blood loaded with oxygen and blood lacking oxygen. The amount of light transmitted is detected and amplified. And then signals are produced for each wavelength. Here, these signals are separated 2 parts. One is the wavelength ratio of red light LED and infrared light LED measurement. Wavelength amount of measurement is calculated by the HPF (High Pass Filter), Signal Conditioning and PPG. This ratio is converted to $\mathrm{SpO} 2$ Data Transmitter. The other one is converted to heart (pulse) rate by $2^{\text {nd }}$ Derivative, Signal Conditioning and SD PPG.

Oximeter and Heart (Pulse) Rate block diagram is shown in Figure 3.

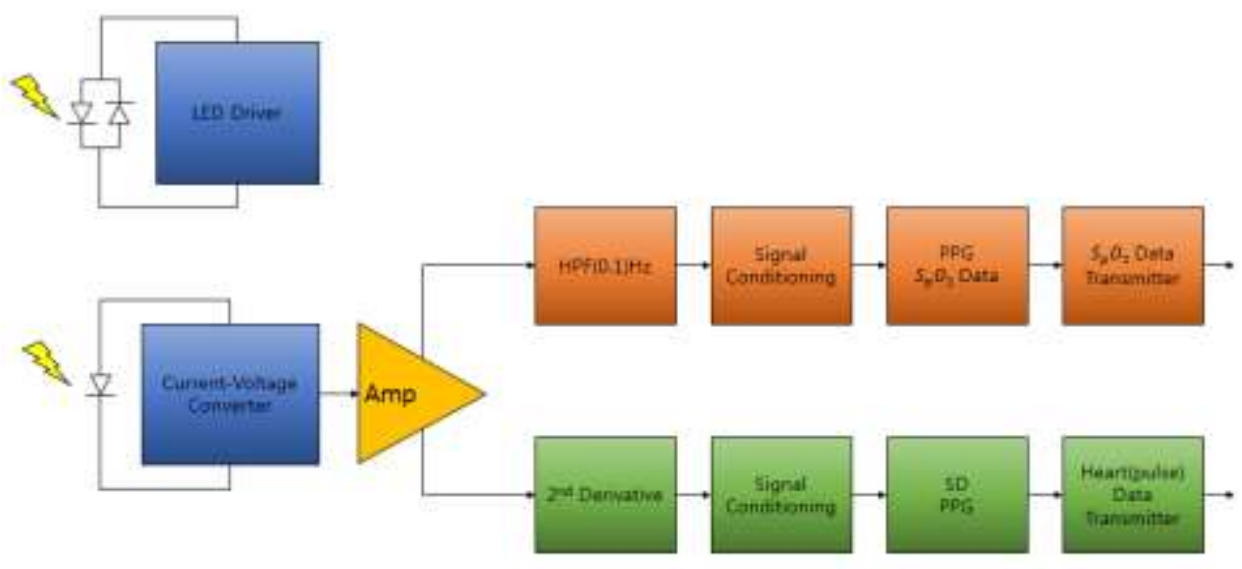

Figure 3. Oximeter and Heart (Pulse) Rate Block Diagram

\subsection{Body Temperature}

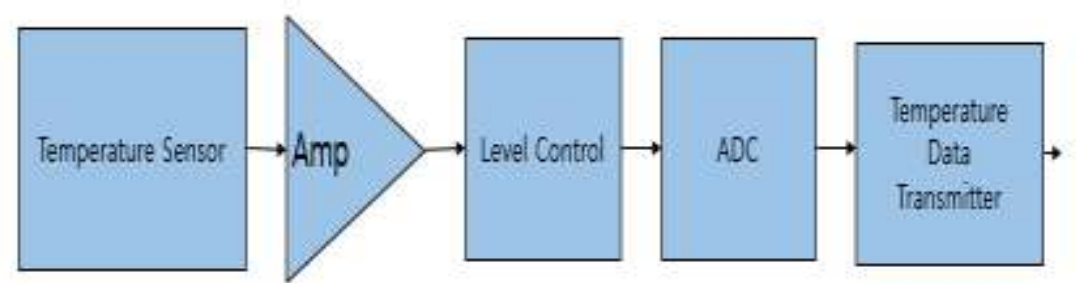

Figure 4. Body Temperature Block Diagram 
Body Temperature block diagram is shown in Figure 4.

Normal human body temperature depends on the age, sex, time of day. Generally typical values are well established $36.8 \pm 0.4^{\circ} \mathrm{C}[1]$

Temperature sensor uses a NTC thermistor type and converted a current to a voltage. After converting ADC, this voltage is divided by the level controller and then temperature data is transmitted.

\subsection{Digital Stethoscope}

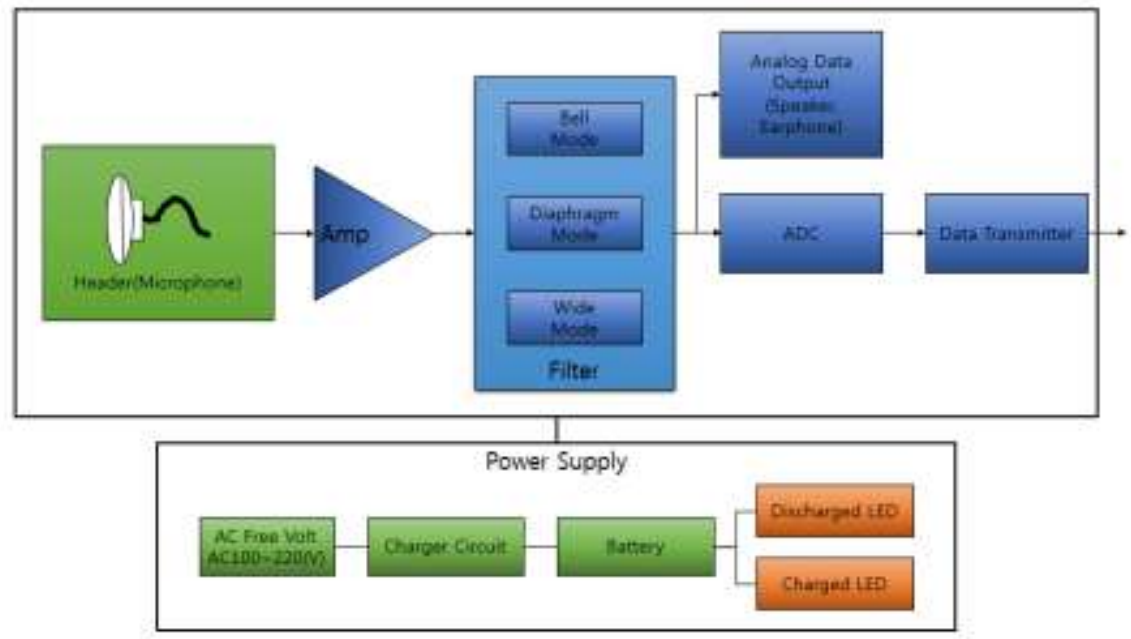

Figure 5. Digital Stethoscope Block Diagram

Digital Stethoscope block diagram is shown in Figure 5. Heart and lung sounds are acquired on the chest by air-coupled microphones (Header). The microphone signals are pre-amplified and low pass filtered (3rd order $1200 \mathrm{~Hz}$ ) and then send to the input interface which includes, automatic gain control, sample and holder and analog to digital converter. A sequence generates the required control signals from a digitally controlled clock [2].

The system is based on a data acquisition boards (VICM). The board has one channel analog inputs which are sequentially digitalized with 12 bit resolution during the sampling period. The board also has digital I/O ports which are used to control the clock frequency and the gain of the amplifiers. The digitalized signals are transferred to the microprocessor memory by wireless (BT).

The following procedure was used to calculate the average level crossing rate. First, the microphone was calibrated independently from the system by using an audio analyzer. The mean sensitivity of a microphone was $12 \pm 3 \mathrm{mV} / \mathrm{Pa}$. Their frequency bandwidth was flat from 20 to $2 \mathrm{KHz}$ by cut off frequency $\pm 3 \mathrm{~dB}$. The analog module was calibrated by applying a $1 \mathrm{mV}$ sinusoidal signal to the inputs of all microphone pre-amplifiers and by storing the corresponding A/D output values for 10 gain levels. And the transfer function was approximated by a linear feedback of these values. The feedback coefficients and the microphone sensitivity of each input were saved in a memory file. During the data acquisition, each heart and lung sounds data is calculated by an average LCR.

In this paper, I suggest LCR algorithm is a versatile technique and has been used widely in signal processing. It is of particular interest in heart and lung sounds processing, because it can be used to calculate spectral correlation. Usually one pitch period of a heart and lung waveform is used as the unit in speech synthesis by the editing of speech elements. Especially level cross processing is explained here because it is the simplest 
case. In this system, one pitch period of heart and lung sounds waveform is stored as a signal element in the form of level cross interval sequence. Therefore, level crossing rate is

$$
\begin{gathered}
\mathrm{L}(\mathrm{m})=\frac{1}{N} \sum_{n=m-\mathbb{N}+1}^{m}\left(\frac{\left|\operatorname{sgn}\left[x(n)-L_{t h}\right]-\operatorname{sgn}\left[x(n-1)-L_{t h}\right]\right|}{2}\right) w(m-n) \\
\operatorname{sgn}[x(n)]=\left\{\begin{array}{cc}
1 & x(n) \geq 0 \\
-1 & x(n)<0
\end{array}\right.
\end{gathered}
$$

These transitional elements are generated by various combinations of the first and second formants. Each of these elements is represented by level cross sequences, on the average, and every interval of level cross has been quantized by 12 bits.

\section{Constructional System}

\subsection{Hardware}

It is experimented under condition such as following articles.

Rating Voltage: DC 5V, 30mA.

Consumption Power: 0.15VA.

Frequency: $0 \sim 2,000 \mathrm{~Hz}$ (Sampling frequency: $4 \mathrm{KHz}$ ).

Gain: 10times amplification.

Electric protecting type \& protecting level: 1st class equipment, B type or BF type equipment.

HEAD microphone (CMIC-9767, 64dB, 9.7*6.7mm)

Earphone (XE-1600)

Processor: ATMEGA16UW-MUR

A picture of VICM diagnosis hardware system is shown in Figure 6. Name of each part is as following. This is a front side.

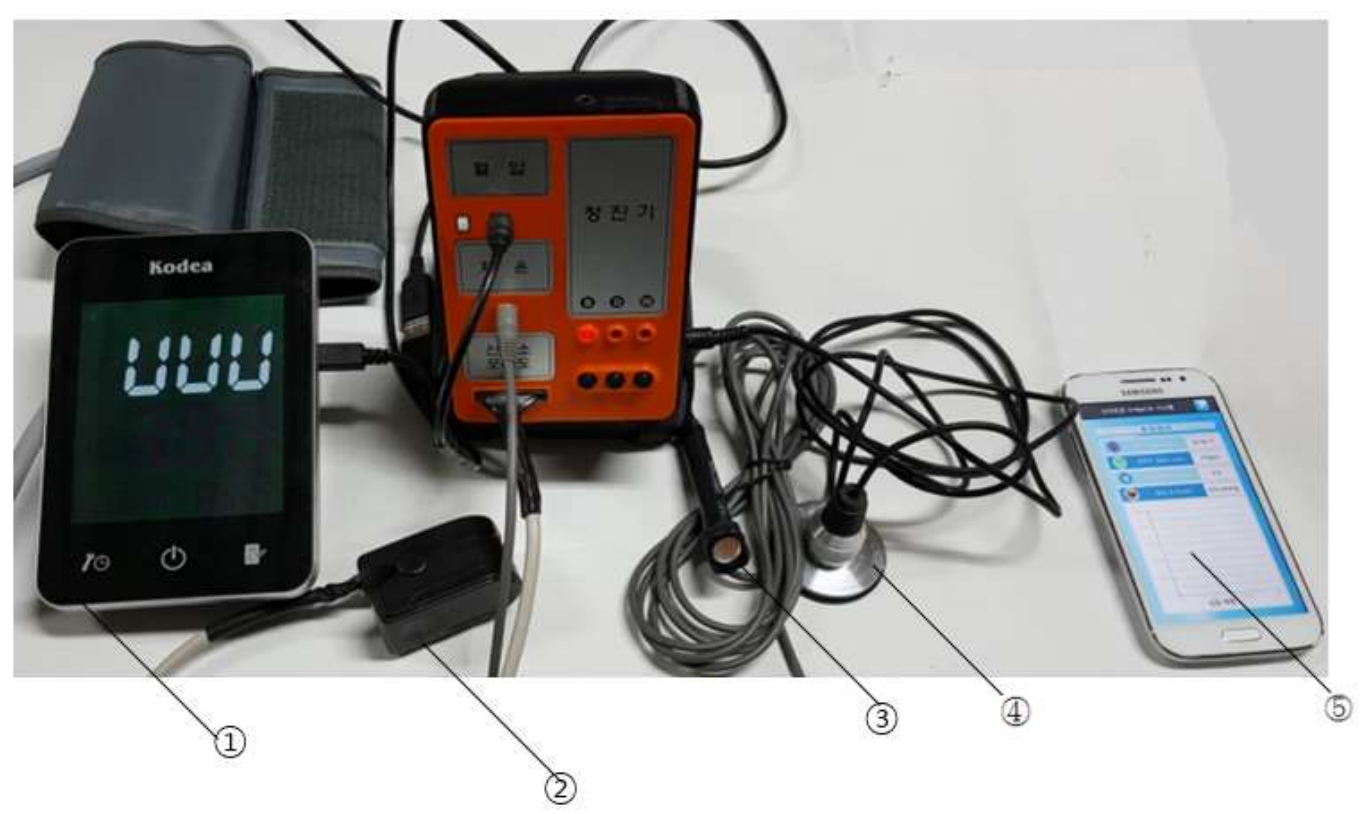

Figure 6. VICM Diagnosis System 
(1) Sphygmomanometer: blood pressure (BP) is the pressure of circulating blood on the walls of blood vessels.

(2) Oximeter: Pulse oximetry is a noninvasive method for monitoring a person's oxygen saturation $\left(\mathrm{SpO}_{2}\right)$.

(3) Body Temperature: Normal human body temperature depends on the age, sex, time of day. Generally typical values are well established in $36.8 \pm 0.4^{\circ} \mathrm{C}$ [1].

(4) Digital Stethoscope: Digital Stethoscope diagnoses heart and lung sounds of human.

5 Smartphone: It displays the record of the four medical devices by the App. program.

\subsection{Software}

The App. program of the smartphone diagnosis software graphics is shown in Figure 7. It displays the record of the four medical devices (body temperature, heart (pulse) rate, $\mathrm{SpO}_{2}$, blood pressure and heart (lung) sound waveform) by the smartphone diagnosis App.

A biomedical diagnosis algorithm is installed in the smartphone in order to process the heart and lung sound data collected by the header (microphone). LCR (level crossing rate) of each biomedical signal is analyzed using a predetermined biomedical signal analysis algorithm included in a LCR calculation, and is displayed on a smartphone window.

And in order to extract the characteristics of heart and lung sound signals, acoustic parameters are required for the analysis of signals. And the heart and lung sound signals have information of human disease on the time based. So the digitization of the vital sounds data is required in order to overcome the limitation of hardware resource. Also, the lossless compression of heart and lung sound information is required.

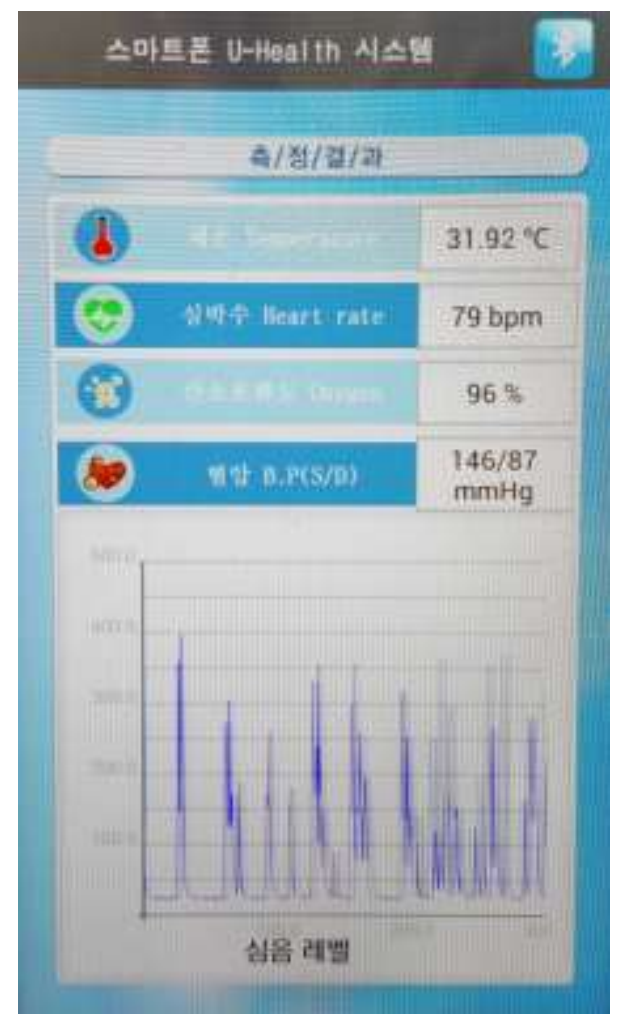

Figure 7. Smartphone App. Program

All of the numerical values are displayed on the smartphone window. The monitor is implemented using a touch screen, so that the operation of the monitor is easy, and thus, there is an advantage of shortening diagnosis time.

Since the level crossing rate is calculated by the logarithmic energy level crossing rate calculation in the microprocessor using Equation (1). [4] 
$\mathrm{Lm}$ is a threshold value and is determined by the characteristic of the input signal and various examination results. Since the determination of the threshold value and a large amount of calculation are problems, it is assumed that the value of Lm is not always constant, and the value of $\mathrm{Lm}$ is determined by observing variation in the characteristics of the input signal and using the average characteristic of the input signal. That is, the threshold value is determined by input variables. Accordingly, the threshold value which is experimentally used for the detection of signals is fixed, so that a threshold value that varies depending on the situation is determined, a biomedical signal interval is detected by performing FFT on sound data and using the band of frequency $20 \mathrm{~Hz} \sim 2 \mathrm{kHz}$ by the LCR.

As a result, there is an advantage in that data loss and the diagnosis of a patient's disease and condition according to subjective comments based on the hearing ability of a physician. It is possible to perform an objective medical diagnosis thanks to visual confirmation and determination.

\section{Results and Discussion}

Figure 7 shows the heart sound level of healthy person in the window of smartphone. Here, the constant pitch and interval of the heart sound level indicate a healthy heart condition.

And the body temperature, blood pressure, oxygen saturation, pulse rate and heart (lung) sounds are displayed in the window of smartphone. In other words, it is possible to accurately diagnose a person's health condition by measuring vital sign. All data of VICM diagnosis system has collected to use analysis software by making visual $\mathrm{C}++$. Table 1 shows an example of a program used for blood pressure measurement. 


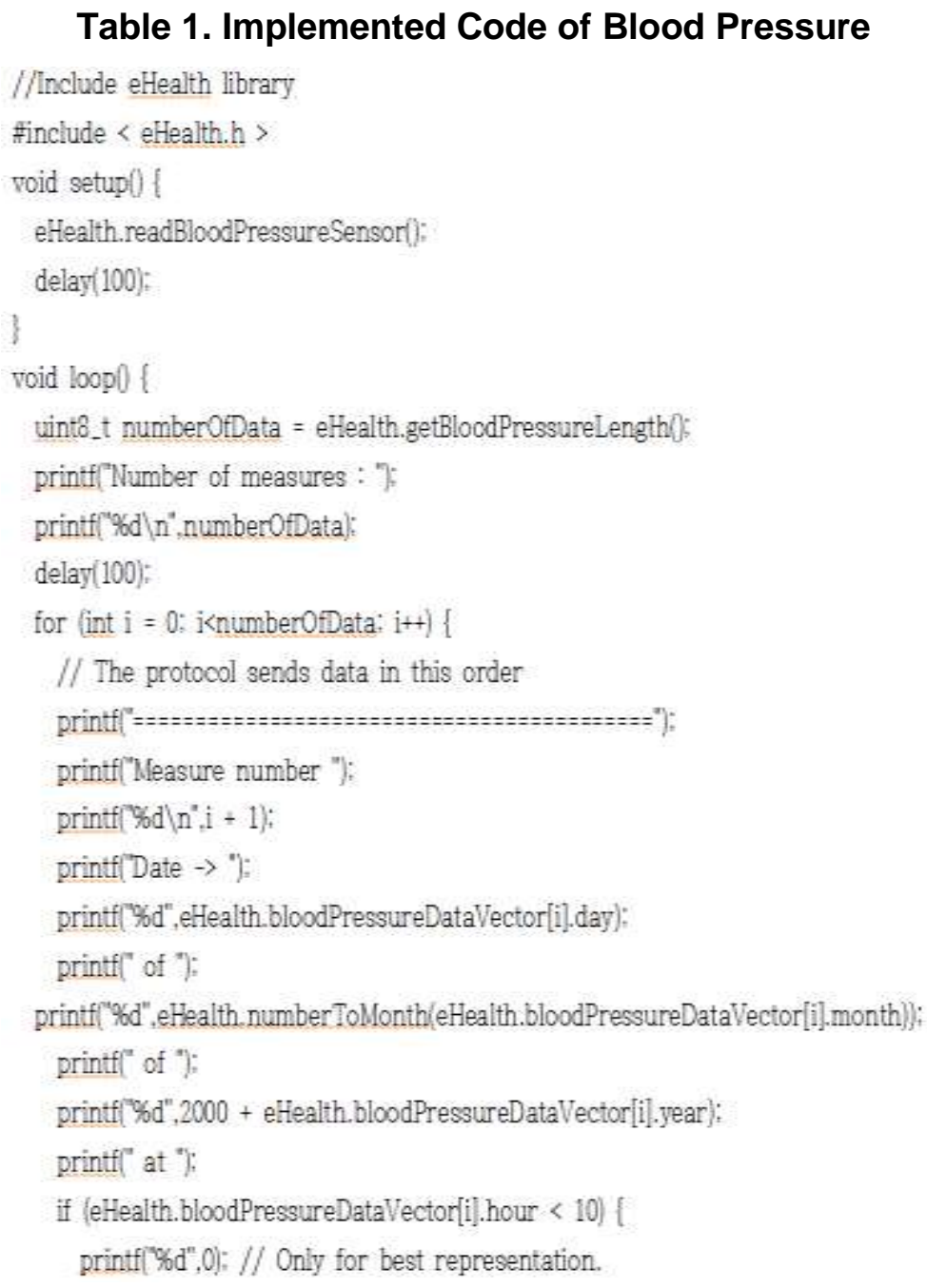

Therefore, we could discriminate between normal and abnormal case using level crossing rate. Figure 8 shows the result of the test by a KTC (Korean Testing Certification). The VICM diagnosis system has been designed with sphygmomanometer, oximeter, body temperature and a stethoscope terminal and microprocessor controls the terminal. Let's check the results of the test results for each system. The accuracy of the sphygmomanometer for pressure 50,100,150,200, 250 and $300(\mathrm{mmHg})$ is less than 2 percent. So, it can be confirmed that the proposed blood pressure monitor can be used as a medical device. The reference value of oxygen saturation for $70,80,90$ and 100 percent is less than one percent. Also, it can be confirmed that the proposed oximeter can be used as a medical device. 

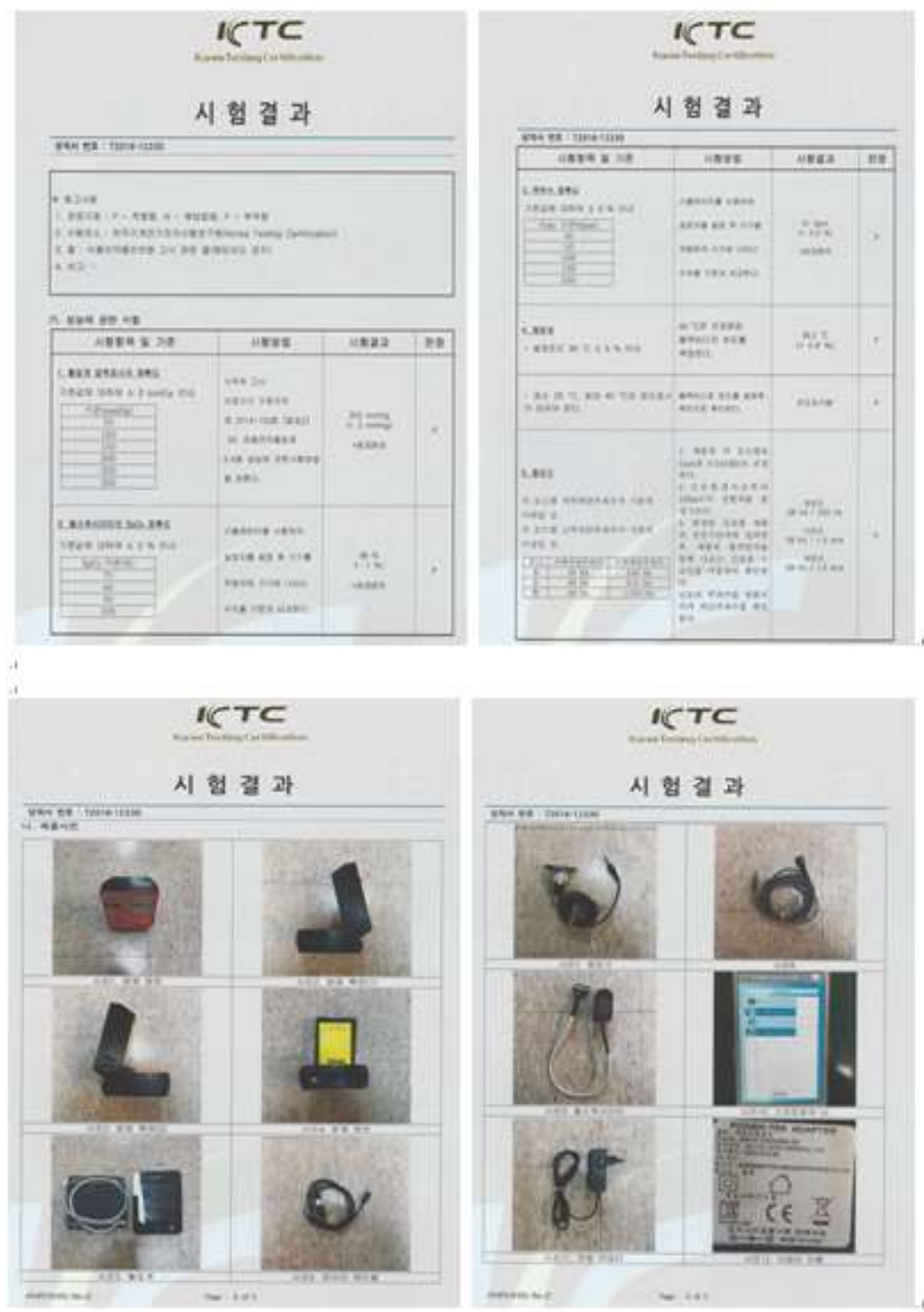

Figure 8. Test Result of KTC.

The reference values of heart (pulse) rate at 30, 50, 100, 150 and 250 (bpm) is less than 3.3 percent. So, it can be confirmed that the proposed heart (pulse) rate can be used as a medical device. The set of body temperature is at 36.2 degrees, and the minimum temperature is at 30 degrees to maximum 40 degrees. The result of the set temperature (36.2) accuracy of the thermometer is within 0.6 percent.

The stethoscope has three modes which are bell, diaphragm and wide. Bell mode is used to hear heart sounds. The range of the bell mode frequency is set from $28(\mathrm{~Hz})$ to $253(\mathrm{~Hz})$. Diaphragm mode is used to hear lung sounds. The range of the diaphragm mode frequency is set from $28(\mathrm{~Hz})$ to $600(\mathrm{~Hz})$. However, the high frequency of diaphragm 
mode comes to $1,200(\mathrm{~Hz})$. This is a problem in diagnosing lung sound. We think that the high pass filter should be redesigned. Wide mode is used to hear heart and lung sounds. The range of the wide mode frequency is set from $28(\mathrm{~Hz})$ to $1,200(\mathrm{~Hz})$.

Heart and lung sounds of patients are acquired on the chest by air-coupled header. Header is consisted with condenser and dynamic microphone. In other words, header will be adapted with various sound collectors. The header is shown in Figure 9.

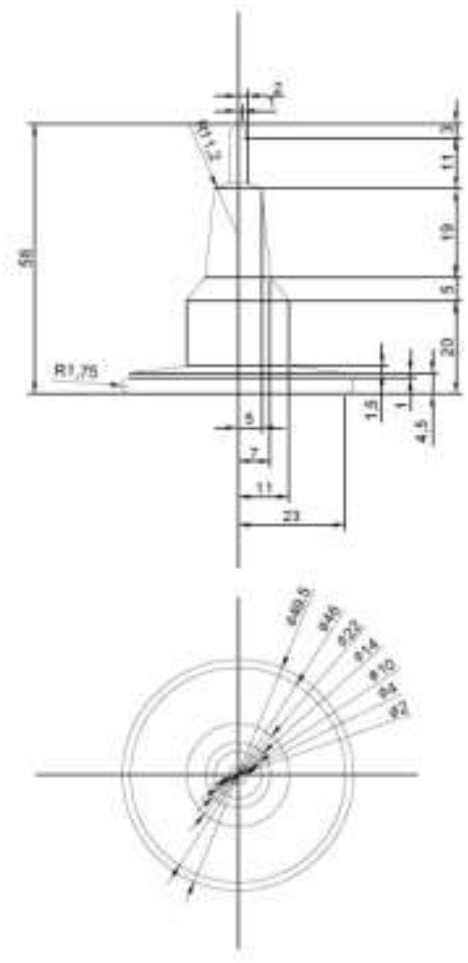

Figure 9. Header Block Diagram of the Stethoscope

\section{Conclusion}

In the experiment for vital sign (body temperature, blood pressure, oxygen saturation, pulse rate, heart and lung sound), we introduced new diagnostic system using VICM hardware and graphic design with app. programming. This paper proposed characteristic heart and lung sound to use LCR.

Figure 8 shows various values of vital sign and the numbers indicated the health condition of a person. VICM hardware system is shown in Figure 6.

Considering the above results, it is necessary to study for more accurate hardware structure and software of the VICM system.

On the other hand, a smartphone u-healthcare diagnosis system using VICM is constructed and we have developed a convenient smartphone diagnostic system that can diagnose a person's health condition anytime and anywhere without being restricted by place and time. 


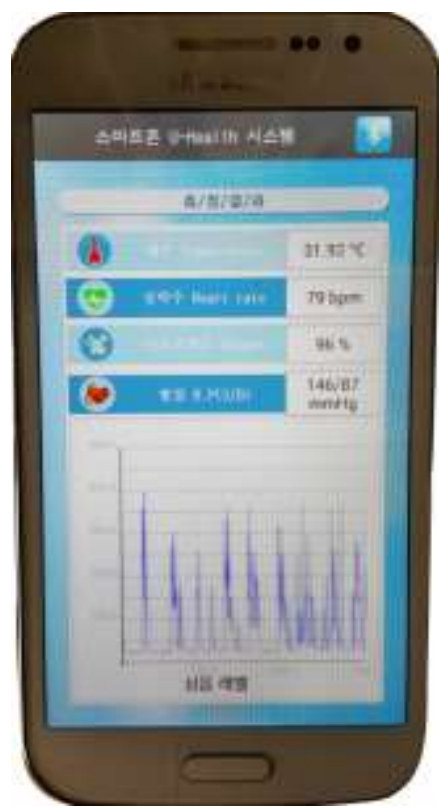

\section{Figure 10. Smartphone u-Health System}

\section{Acknowledgments}

This paper is a revised and expanded version of a paper entitled [The $3^{\text {rd }}$ International Mega-Conference on Green and Smart Technology] presented at [Eui-Bung Jeong, Soomi Hong, Kee-young Park, Jeju National University, Jeju, Korea, and Dec. $21^{\text {st }} 2016$.].

\section{References}

[1] http://en.wikipedia.org/wiki/Blood_pressure

[2] K.Y. Park, "Hardware Construction of Visual Diagnosis System," KICS, Honam Section, vol. 15, no.1, (2006), pp11-17.

[3] K.Y. Park, "Wireless Diagnosis System for Chest Disease using Thoracic Sound," IEEE, Honam Section, vol. 13, no.1, (2004), pp57-62,

[4] K.Y. Park, S.M. Hong, J.H. Lee, J.H. Park and E.B. Jung, "Animal Diagnosis System using Wireless Digital Stethoscope.” KICS vol. 38B, no. 09, (2013), pp722-727.

[5] A.J. Robertson, C. R. Rales, L. Rhonchi, Lancet, (1957), pp417-23.

[6] D. W. Cugell, "Lung Sound Nomenclature" Northwestern Univ. Medical School, Chicago, Illinois, (1987).

[7] E.B. Jeoung, S.M. Hong and K.Y. Park, "Ubiquitous Healthcare Diagnosis System for Smartphone using Vital Integrated Medical Instrument", Proceedings of the $3^{\text {rd }}$ International Mega-Conference on Green and Smart Technology, (2016); Jeju Island, Korea.

\section{Authors}

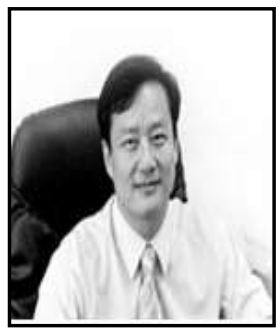

Eui-Bung Jeoung, He received the $\mathrm{PhD}$ degree in Electronic Engineering from Konkuk University, Korea, in 1992. He us a professor at Automotive \& Mechanical Engineering Department, Howon University. His research interests include Digital signal processing, and IoT. 


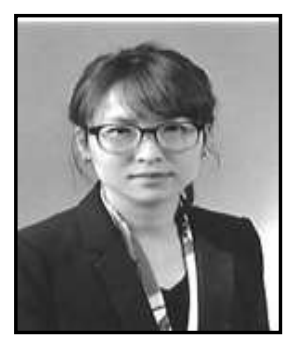

Soomi Hong, She received the B.S. degree in public health administration from purdue University, USA in 2013. Currently she is studying for the $\mathrm{PhD}$ degree in English language and culture department. She is an associate professor at public health administration department, Jeonju Vision University. Her research interests include U-Healthcare system, and IoT.

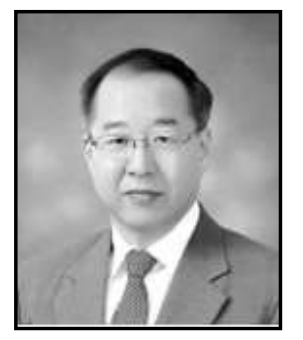

Kee-young Park, he received the $\mathrm{PhD}$ degree in Electronic Engineering from Jeonbuk University, Korea, in 2002. He is a professor with Digital Electronics \& Information, Jeonju Vision University. His research interests include Signal processing, and BioMedical Processor system. 